Accepted for publication in Physics Letters B

\title{
Transition from Thermal to Rapid Expansion in Multifragmentation of Gold Induced by Light Relativistic Projectiles
}

\author{
S.P. Avdeyev ${ }^{a}$, V.A. Karnaukhov ${ }^{a}$, L.A. Petrov ${ }^{a}$, \\ V.K. Rodionov ${ }^{a}$, V.D. Toneev ${ }^{\mathrm{a}}$, H. Oeschler ${ }^{\mathrm{b}}$, \\ O.V. Bochkarev ${ }^{c}$, L.V. Chulkov ${ }^{\mathrm{c}}$, E.A. Kuzmin ${ }^{\mathrm{c}}$, \\ A. Budzanowski ${ }^{d}$, W. Karcz ${ }^{d}$, M. Janicki ${ }^{d}$, E. Norbeck ${ }^{\mathrm{e}}$, \\ A.S. Botvina ${ }^{f}$ \\ ${ }^{a}$ Joint Institute for Nuclear Research, 141980 Dubna, Russia \\ b Institut für Kernphysik, Technische Universität Darmstadt, D-64289 Darmstadt, \\ Germany \\ ${ }^{\mathrm{c}}$ Kurchatov Institute, 123182, Moscow, Russia \\ ${ }^{\mathrm{d}}$ H. Niewodniczanski Institute of Nuclear Physics, 31-342, Cracow, Poland \\ ${ }^{\mathrm{e}}$ University of Iowa, Iowa City, IA 52242 USA \\ ${ }^{\mathrm{f}}$ Institute for Nuclear Research, 117312, Moscow, Russia
}

\begin{abstract}
Multiple emission of intermediate-mass fragments has been studied for the collisions of $\mathrm{p},{ }^{4} \mathrm{He}$ and ${ }^{12} \mathrm{C}$ on $\mathrm{Au}$ with the $4 \pi$ setup FASA. In the case of ${ }^{12} \mathrm{C}(22.4 \mathrm{GeV})+\mathrm{Au}$ and ${ }^{4} \mathrm{He}(14.6 \mathrm{GeV})+\mathrm{Au}$ collisions, the deviations from a pure thermal break-up are seen in the energy spectra of the emitted fragments: the spectra are harder than calculated and than measured in p-induced collisions. This difference is attributed to a collective flow with the expansion velocity on the surface about $0.1 c$ (for ${ }^{12} \mathrm{C}+\mathrm{Au}$ collisions).
\end{abstract}


Nuclear multifragmentation is a new decay mode of excited nuclei characterized by the emission of Intermediate Mass Fragments (IMF, $3 \leq Z \leq 20$ ). The development of this field has been strongly stimulated by the idea that this process might be related to a liquid-gas phase transition in nuclear matter. A recent status on multifragmentation can be found in Ref. [1].

Highly excited nuclei can be formed by heavy ion collisions at intermediate energies, but there are advantages in producing the excitation by light relativistic projectiles. In the first case, nuclear heating is accompanied by compression, fast rotation and shape distortion which may cause dynamic effects in the multi-fragment disintegration and it is not easy to disentangle all these effects and extract information on thermodynamic properties of hot nuclear systems. The situation becomes more transparent if light relativistic projectiles are used. In this case, dynamic effects are expected to be negligible. Another advantage is that all the fragments are emitted by a single source: a slowly moving target remainder. Its excitation energy might be almost entirely thermal. Light relativistic projectiles provide therefore an unique possibility to study "thermal multifragmentation". It has been shown that thermal multifragmentation indeed takes place in collisions of light relativistic projectiles $\left(\mathrm{p},{ }^{3} \mathrm{He}, \alpha\right.$ and recently $\pi$ and $\overline{\mathrm{p}}$ ) with a heavy target $[2-12]$.

In this Letter we concentrate on studying energy spectra of the emitted fragments as they reflect, due to the "Coulomb law", the geometry and dynamics (expansion) of the emitting source. By comparing our results from $\mathrm{p}+\mathrm{Au}[4]$ collision with those from reactions induced by $\alpha$ particles and ${ }^{12} \mathrm{C}$ projectiles with incident energies of $(1-4) A \cdot \mathrm{GeV}$, we evidence a transition from a pure statistical process ("thermal multifragmentation") to a behaviour reflecting dynamics. It will be shown that from the observed additional collective energy a spatial distribution of the fragments at freeze out can be infered.

The experiments were performed with beams from the JINR synchrophasotron

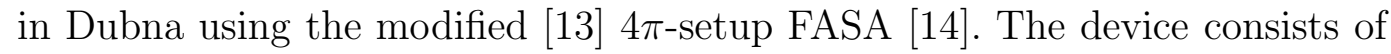
two main parts : (i) Five $\Delta E$ (ionization chamber) $\times E(\mathrm{Si}$ )-telescopes, which serve as a trigger for the read-out of the system allowing measurement of the charge and energy distributions of IMF's at various angles from $24^{\circ}$ to $156^{\circ}$ covering together a solid angle of $0.03 \mathrm{sr}$. (ii) The fragment multiplicity detector consisting of $64 \mathrm{CsI}(\mathrm{Tl})$ counters (with thicknesses around $30 \mathrm{mg} \cdot \mathrm{cm}^{-2}$ ) which covers $89 \%$ of $4 \pi$. This device gives the number of IMF's in the event and their spatial distribution. A self-supporting Au target of $1.5 \mathrm{mg} / \mathrm{cm}^{2}$ thickness was located in the center of the FASA vacuum chamber $(\sim 1 \mathrm{~m}$ in diameter). The following beams were used: protons at energies of 2.16, 3.6 and $8.1 \mathrm{GeV}$, ${ }^{4} \mathrm{He}$ at energies of 4 and $14.6 \mathrm{GeV}$ and ${ }^{12} \mathrm{C}$ at $22.4 \mathrm{GeV}$. The average beam intensity was $7 \cdot 10^{8}$ particles/spill for protons and helium and $1 \cdot 10^{8}$ particles/spill for carbon projectiles with a spill length at $300 \mathrm{~ms}$ and a spill period of $10 \mathrm{~s}$. See also [4] reporting on the $\mathrm{p}+\mathrm{Au}$ experiment. 
The kinetic energy of fragments is determined by four terms: thermal motion, Coulomb repulsion, rotation, and collective expansion energies of the system at freeze out, $E=E_{t h}+E_{C}+E_{\text {rot }}+E_{\text {flow }}$. The additivity of the first three terms is quite obvious. For the last term, its independence of others may be considered only approximately when the evolution of the system after the freeze-out point is driven only by the Coulomb force. The Coulomb term is significantly larger than the thermal one as was shown in Ref. [7] for ${ }^{4} \mathrm{He}$ $(14.6 \mathrm{GeV})+\mathrm{Au}$ collisions, where the Coulomb part of the mean energy of the carbon fragment is three times larger than thermal one using volume emission of fragments from a diluted system.

The contribution of the collective flow for the $\mathrm{p}+\mathrm{Au}$ collisions at $8.1 \mathrm{GeV}$ incident energy was estimated to $v_{\text {flow }}<0.02 c$ in Ref. [4]. This was done by comparing the measured IMF spectra with the ones calculated within the framework of the Statistical Multifragmentation Model (SMM) [15] which includes no flow. For heavy ion collisions, collective flow has been observed and it is the most pronounced in central $\mathrm{Au}+\mathrm{Au}$ collisions [16]. In this respect, it is interesting to analyse energy spectra of fragments from $\mathrm{He}+\mathrm{Au}$ and $\mathrm{C}+\mathrm{Au}$ collisions looking for a possible onset of collective flow phenomenon.

A comparison of the energy spectra of outgoing carbon isotopes from proton-, helium- and carbon-induced collisions on a $\mathrm{Au}$ target is presented in Fig. 1. The spectral shapes show an increase in the number of high-energy carbon fragments with the projectile mass.

The reaction mechanism for light relativistic projectiles is usually divided into two stages. The first one is a fast energy-depositing stage, during which very energetic light particles are emitted and a nuclear remnant (target spectator) is excited. We use a refined version of the intranuclear cascade model (INC) [17] for describing the first stage. The second stage is described by the Statistical Multifragmentation Model (SMM), which considers multibody decay (volume emission) of a hot expanded nucleus. But such a two-stage approach fails to describe the observed IMF multiplicities as shown in Table 1. An expansion stage is inserted between the two parts of calculation. The excitation energies and the residual masses are then fine tuned [4] to get an agreement with the measured IMF multiplicities, i.e. the values for the residual (after INC) masses $A_{R}$ and their excitation energies $E_{R}$ are scaled on an event-by-event basis. The average masses of nuclei which decay by multifragmentation is labelled $A_{M F}$ having mean excitation energies $E_{M F}$ as given in Table 1 together with the values of $\left\langle M_{I M F}\right\rangle$. The lines in Fig. 1 give the spectra calculated in the framework of this combined model INC+Expansion+SMM. The fragment energies are obtained by the multibody Coulomb trajectory calculations on an event-by-event basis. In the initial state all the charged particles are assumed to have a thermal velocity only (no flow). 
The calculated carbon spectrum for $\mathrm{p}+\mathrm{Au}$ collisions (at $8.1 \mathrm{GeV}$ ) is consistent with the measured one. A similar situation occurs with ${ }^{4} \mathrm{He}+\mathrm{Au}$ collisions at $4 \mathrm{GeV}$, but not with ${ }^{4} \mathrm{He}(14.6 \mathrm{GeV})+\mathrm{Au}$ and ${ }^{12} \mathrm{C}+\mathrm{Au}$ interactions: the measured spectra are harder than the calculated ones. A careful inspection of the reaction induced with $\alpha$ particles shows that the maximum of the energy spectra obtained at $4 \mathrm{GeV}$ is higher than obtained at $14.6 \mathrm{GeV}$. This is mainly caused by the higher $Z$ values of the residual nuclei at the lower incident energy and is reproduced by the model calculations. The slope of the 14.6 $\mathrm{GeV}$ data is much harder than for the lower incicent energy. This higher slope is underestimated by the model and this failure is most pronounced for $\mathrm{C}+$ $\mathrm{Au}$ collisions. An attempt to describe these spectra by a higher Coulomb field, caused e.g. by a more compact system at break-up fails. In such an approach the maximum shifts keeping the drop towards higher kinetic energies while the measured spectra show an increase at the higher kinetic energies without moving the maximum significantly.

The trends from Fig. 1, i.e. increasing mean energies with increasing mass and increasing energy of the projectile, is seen for many emitted fragments. This observation is summarized in Fig. 2, which shows the mean kinetic energies per fragment nucleon as a function of the charge of the detected fragments $Z$. This figure shows a remarkable enhancement in the kinetic energies for light fragments emitted in $\mathrm{He}+\mathrm{Au}$ and $\mathrm{C}+\mathrm{Au}$ collisions as compared to the $\mathrm{p}(8.1$ $\mathrm{GeV})+\mathrm{Au}$ case. The calculated values of the mean fragment energies (shown by lines) are obtained with INC+Expansion+SMM model. The measured energies are close to the calculated ones for $\mathrm{p}+\mathrm{Au}$ collisions in the range of the fragment charges between 4 and 9 . The experimental values for ${ }^{4} \mathrm{He}+\mathrm{Au}$ and ${ }^{12} \mathrm{C}+\mathrm{Au}$ interactions, however, exceed the calculated ones, which are similar for all three cases.

The observed deviation cannot be attributed to an angular momentum effect. The rotational energy $E_{\text {rot }}$ of a fragment with mass $A_{I M F}$ can be estimated from the total rotational energy $E_{L}$ of a system with mass number $A_{R}$ using classical rotation:

$$
<E_{\text {rot }}>/ A_{I M F}=\frac{5}{3}<\frac{E_{L}}{A_{R}}>\frac{<R_{Z}^{2}>}{R_{\text {sys }}^{2}}
$$

where $R_{Z}$ is the radial coordinate of the IMF and $R_{\text {sys }}$ is the radius of the system. According to the INC calculations for $\mathrm{C}+\mathrm{Au}$ collisions, the mean angular momentum $L$ of the target spectator is $\approx 36 \hbar$. It might be reduced by a factor of 1.5 due to the mass loss during expansion along the way to the freeze-out point. Finally $\left\langle E_{L}\right\rangle$ is estimated to be only $5 \mathrm{MeV}$ and $<E_{\text {rot }}>/ A_{I M F} \approx 0.04 \mathrm{MeV} /$ nucleon, which is by an order of magnitude smaller than the energy enhancement for light fragments. We suggest that the observed enhancement is caused by the expansion of the system, which 
seems to be radial, as a $v_{\|}$-versus- $v_{\perp}$ plot (this will be subject of a forthcoming publication) does not show any significant deviation from circular symmetry.

An estimate of the fragment flow energy may be obtained from the difference between the measured IMF energies $\langle E\rangle$ and those calculated (without any flow $)<E>_{\text {noflow }}$. This difference for $\mathrm{C}+\mathrm{Au}$ collisions is shown in the lower part of Fig. 2. A drastic decrease of the flow energy with increasing charge of the fragment is seen.

In an attempt to describe the data, we modified the SMM code by including a radial velocity boost for each particle at freeze out, i.e. a radial expansion velocity was superimposed on the thermal motion in the calculation of the multibody Coulomb trajectories on an event-by-event basis. A self-similar radial expansion of a spherical nucleus is assumed, where the local flow velocity is linearly dependent on the distance of the particle from the centre of mass. The expansion velocity of particle $Z$ located at radius $R_{Z}$ is given by the following expression

$$
\vec{v}_{\text {flow }}(Z)=v_{\text {flow }}^{0} \cdot \frac{\vec{R}_{Z}}{R_{\text {sys }}}
$$

where $v_{\text {flow }}^{0}$ is the radial velocity on the surface of the system. The use of the linear profile of the radial velocity is motivated by the hydrodynamic models for an expanding hot nuclear system (see for example Ref. [18]). The value of $v_{\text {flow }}^{0}$ has been adjusted to $0.1 c$ in order to describe the mean kinetic energy measured for the carbon fragments. The results are presented in the lower part of Fig. 2 as dashed line calculated from the difference of the fragment energies obtained for $v_{\text {flow }}^{0}=0.1 c$ and $v_{\text {flow }}^{0}=0$. The data deviate significantly from the calculated values for $\mathrm{Li}$ and Be. This may be caused in part by the contribution of particle emission during the early stage of expansion from the hotter and denser system. It is supported by the fact that the extra energy of $\mathrm{Li}$ fragments with respect to the calculated value is clearly seen in Fig. 2 even for the proton-induced fragmentation, where no significant flow is expected. This peculiarity of light fragments has been noted already by the ISIS group for ${ }^{3} \mathrm{He}+\mathrm{Au}$ collisions at $4.8 \mathrm{GeV}[10]$.

For fragments heavier than carbon, the calculated curve in the lower part of Fig. 2 is higher than the data and only slightly decreasing with increasing fragment charge. The trend of the calculation is to be expected. The mean fragment flow energy is proportional to $\left\langle R_{Z}^{2}>\right.$ and this value changes only little with fragment charge in the SMM code due to the assumed equal probability of IMF distribution inside the available break-up volume. The difference between data and calculations shown in Fig. 2 indicates that a uniform density distribution is not fullfilled. A preference of finding heavier fragments more in the center would reduce their Coulomb energies shown in the upper part of Fig. 2, 
and would increase the extracted quantity $<E^{\text {measured }}>-<E>_{\text {noflow }}$ leading then to a consistent description of a flow scenario with a non-uniform fragment distribution. The dense interior of the expanded nucleus may be favored for the appearance of larger IMF's, if fragments are formed via the density fluctuations. Indications for such an effect could already be drawn from the analysis of the mean IMF energies performed in Ref. [4] for proton induced fragmentation: the measured energies are below the theoretical curve for fragments heavier than Ne. This means that our procedure of estimating the flow energy is only reliable for fragments lighter than Ne where the model calculations fit the data of the fragment energies from $\mathrm{p}+\mathrm{Au}$ collisions (no flow). For heavier fragments the flow energies are underestimated and should be considered as lower limits. The interesting feature of a reduction of the flow energy for heavier fragments is observed also for the central heavy ion collisions [19]. This effect is increasingly important at energies $\leq 100 \mathrm{~A} \cdot \mathrm{MeV}$, and that is in accordance with our suggestion on its relation to the density profile of the hot system at freeze out.

For the estimation of the mean fragment flow velocities $\left\langle v_{\text {flow }}>\right.$ the difference between the measured IMF energies and calculated ones (without flow) has been used. The results are presented in Fig. 3. The values for $\mathrm{Li}$ and Be are considered as upper limits because of the possible contribution of the preequilibrium emission. The corresponding values of the fragment positions $<R_{Z}>/ R_{\text {sys }}$ for the freeze-out point obtained under assumptions of the linear radial profile for the expansion velocity are given on the right-hand scale of this figure. The dotted line shows the mean radial coordinates of fragments according to the SMM code. As it has been noted above, the calculated values of $\left\langle R_{Z}\right\rangle / R_{\text {sys }}$ are only slightly decreasing with $Z$ as expected from a uniform density distribution, but in clear contrast to the data. Calculations with the MMMC model [20] give the same trend as the SMM model [21].

Effects of the radial collective energy for $1 A \cdot \mathrm{GeV} \mathrm{Au}+\mathrm{C}$ collisions (in inverse kinematics) were considered in [22] by analysing the transverse kinetic energies. The mean radial flow velocities were estimated, but it had been done only for fragments with $Z \leq 7$. In this charge range our analysis gives slightly lower values of the mean expansion velocities as compared to Ref. [22]. Their interpretation of a time sequence of the emission acccording to the $Z$ value is largely equivalent to our interpretation.

The total expansion energy can be estimated by integrating the nucleon flow energy (taken according to Eq. (2)) over the available volume at freeze out. For an uniform system one obtains

$$
E_{\text {flow }}^{\text {tot }}=\frac{3}{10} A_{R} \cdot m_{N}\left(v_{\text {flow }}^{0}\right)^{2}\left(1-r_{N} / R_{\text {sys }}\right)^{5}
$$


with the mass $m_{N}$ and the radius $r_{N}$ of a nucleon. For ${ }^{12} \mathrm{C}+\mathrm{Au}$ collisions it gives $E_{\text {flow }}^{\text {tot }} \simeq 100 \mathrm{MeV}$, corresponding to the flow velocity at the surface of 0.1c. Similar results are obtained for ${ }^{4} \mathrm{He}(14.6 \mathrm{GeV})+\mathrm{Au}$ collisions. The total flow energy of the fragmenting nucleus is four times less than the thermal one estimated in the INC+Expansion+SMM approach.

Concluding, the energy spectra of IMF's for reactions $\mathrm{p}+\mathrm{Au}$ at 2.1, 3.6 and 8.1 $\mathrm{GeV},{ }^{4} \mathrm{He}+\mathrm{Au}$ at 4 and $14.6 \mathrm{GeV},{ }^{12} \mathrm{C}+\mathrm{Au}$ at $22.4 \mathrm{GeV}$ are compared. While the fragment kinetic energies are well described within the SMM code for $\mathrm{p}+\mathrm{Au}$ collisions, the model underestimates the kinetic energies of fragments from collisions induced by ${ }^{4} \mathrm{He}(14.6 \mathrm{GeV})$ and ${ }^{12} \mathrm{C}(22.4 \mathrm{GeV})$ projectiles. The additional energy is attributed to collective expansion. However, a linear flow profile fails to describe the variation of flow energies extracted from the measured spectra with the fragment charge. This discrepancy might be caused by the fact that the model assumes a uniform density distribution and, hence, a rather uniform probability distribution of forming fragments in the freezeout volume. The data indicate that heavy fragments are preferentially located more in the interior of the nucleus.

The presented study shows that in spite of the success of statistical multifragmentation models in describing the partitions, the freeze-out condition might be still too simplified. The energy spectra provide sensitive probes for the source configuration and the emission dynamics. The range of projectiles, from protons to light nuclei, seems to be quite attractive in this respect for showing a transition from "thermal break-up" to a disintegration possibly caused by rapide expansion likely together with a non-uniform density profile of the excited nucleus.

The authors are thankful to Profs. A. Hrynkiewicz, A.M. Baldin, S.T. Belyaev, A.I. Malakhov and N.A. Russakovich for support. The research was supported in part by Grant No 00-02-16608 from Russian Foundation for Basic Research, by Grant No 2P03 12615 from the Polish State Committe for Scientific Research, by Grant No 94-2249 from INTAS, by Contract No 06DA819 with Bundesministerium für Forschung und Technologie, by Grant PST.CLG.976861 from NATO, and by US National Science Foundation.

\section{References}

[1] Proc. of Int. Workshop XXVII on Gross Properties of Nuclei and Nuclear Excitations, Hirschegg, Austria, Jan. 17-23, 1999, Edited by H. Feldmeier, J. Knoll, W. Nörenberg, J. Wambach, GSI, Darmstadt, 1999.

[2] S.J. Yennello et al., Phys. Rev. Lett. 67 (1991) 671.

[3] Bao-An Li, D.H.E. Gross, V. Lips, H. Oeschler, Phys. Lett. B335 (1994) 1. 
[4] S.P. Avdeyev et al., Eur. Phys. J., A3 (1998) 75.

[5] K. Kwiatkowski et al., Phys. Rev. Lett. 74 (1995) 3756.

[6] V. Lips et al., Phys. Lett. B338 (1994) 141.

[7] S.Y. Shmakov et al., Yad. Fiz. 58 (1995) 1735; (Phys. of Atomic Nucl. 58 (1995) 1635).

[8] G. Wang et al., Phys. Rev. C53 (1996) 1811.; G. Wang et al., Phys. Rev. C57 (1998) R2786.

[9] F. Goldenbaum et al., Phys. Rev. Lett. 77 (1996) 1230.

[10] E.R. Foxford et al., Phys. Rev. C54 (1996) 749.

[11] L. Beaulieu et al., Phys. Lett. B463 (1999) 159.

[12] T. Lefort et al., Phys. Rev. C62 (2000) 031604.

[13] S.P. Avdeyev et al., Pribory i Tekhnika Eksper. 39 (1996) 7; (Instr. Exp. Techn. 39 (1996) 153).

[14] S.P. Avdeyev et al., Nucl. Instrum. Meth. A332 (1993) 149.

[15] J. Bondorf et al., Nucl. Phys. A444 (1985) 476; Phys. Rep. 257 (1995) 133; A.S. Botvina, A.S. Iljinov, I.N. Mishustin, Nucl. Phys. A507 (1990) 649; A.S. Botvina et al., Phys. of Atomic Nuclei 57 (1994) 628.

[16] W. Reisdorf et al., Nucl. Phys. A612 (1997) 493; G.D. Kunde et al., Phys. Rev. Lett. 74 (1995) 38.

[17] V.D. Toneev, K.K. Gudima, Nucl. Phys. A400 (1983) 173c; V.D. Toneev et al., Nucl. Phys. A519 (1990) 463c.

[18] J. Bondorf et al., Nucl. Phys. A296 (1978) 320.

[19] W. Reisdorf, H.G. Ritter, Ann. Rev. Nucl. Part. Sci. 47(1997)663.

[20] D.H.E. Gross, Rep. Progr. Phys. 53 (1990) 605.

[21] J. Lauret, private comminucation.

[22] J. Lauret et al., Phys. Rev. C57 (1998) R1051. 


\begin{tabular}{|c|c|c|c|c|c|c|c|c|}
\hline \multirow{2}{*}{$\begin{array}{c}E_{\text {inc }} \\
(\mathrm{GeV})\end{array}$} & \multirow{2}{*}{ Proj } & \multirow{2}{*}{\begin{tabular}{c} 
Exper. \\
\cline { 4 - 7 }
\end{tabular}} & \multicolumn{5}{|c|}{ Calculations } & \multirow{2}{*}{ Model } \\
\cline { 4 - 8 } & & $M_{I M F}$ & $M_{I M F}$ & $A_{R}$ & $A_{M F}$ & $E_{R}$ & $E_{M F}$ & \\
\hline 3.16 & $\mathrm{p}$ & $1.7 \pm 0.2$ & 1.82 & 189 & 185 & 310 & 589 & INC+SMM \\
& & & 1.69 & 188 & 183 & 288 & 564 & with expansion \\
\hline 8.1 & $\mathrm{p}$ & $2.1 \pm 0.2$ & 3.58 & 183 & 175 & 488 & 808 & INC+SMM \\
& & & 2.52 & 187 & 181 & 371 & 676 & INC+SMM \\
& & & 1.89 & 184 & 175 & 282 & 568 & with expansion \\
\hline 4.0 & ${ }^{4} \mathrm{He}$ & $1.7 \pm 0.2$ & 3.89 & 184 & 177 & 484 & 836 & INC+SMM \\
& & & 1.77 & 177 & 161 & 238 & 502 & with expansion \\
\hline 14.6 & ${ }^{4} \mathrm{He}$ & $2.2 \pm 0.2$ & 4.47 & 173 & 159 & 723 & 1132 & INC+SMM \\
& & & 2.19 & 154 & 103 & 183 & 404 & with expansion \\
\hline 22.4 & $12 \mathrm{C}$ & $2.2 \pm 0.3$ & 4.04 & 163 & 153 & 924 & 1216 & INC+SMM \\
& & & 2.17 & 139 & 86 & 207 & 415 & with expansion \\
\hline
\end{tabular}

Table 1

The calculated properties of nuclear remnants from proj $+\mathrm{Au}$ collisions for INC+SMM and INC+Expansion+SMM. The $M_{I M F}$ is the mean number of IMF's for events with at least one IMF and $A_{R}, E_{R}$ are the mean mass number and excitation energy (in $\mathrm{MeV}$ ), respectively, averaged over all inelastic collisions, while the quantities $A_{M F}, E_{M F}$ are averaged only over residues decaying by IMF emission. 


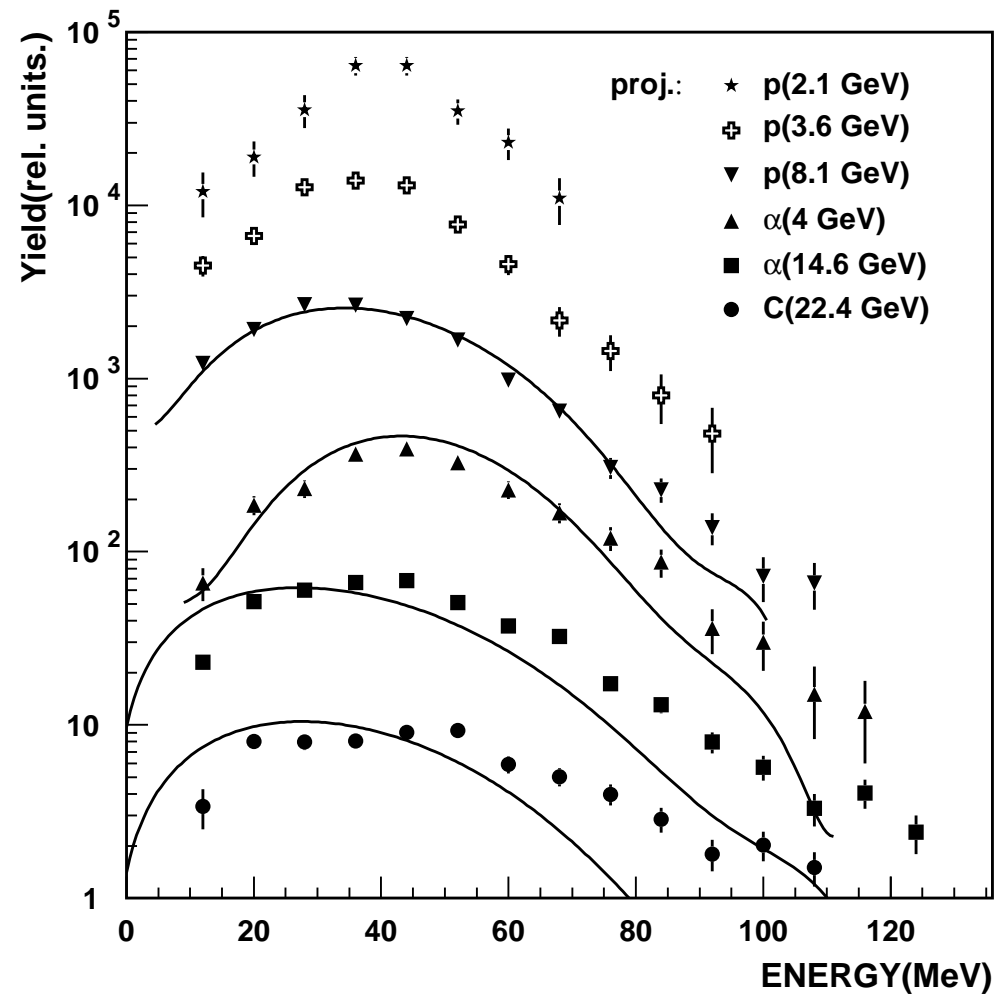

Fig. 1. Energy distribution of carbon fragments obtained for different collision systems at $\theta=89^{\circ}$. The lines are calculated in the INC+Expansion+SMM model assuming no flow. The spectra are arbitrarily scaled along the yield axis to avoid mixing of symbols. 


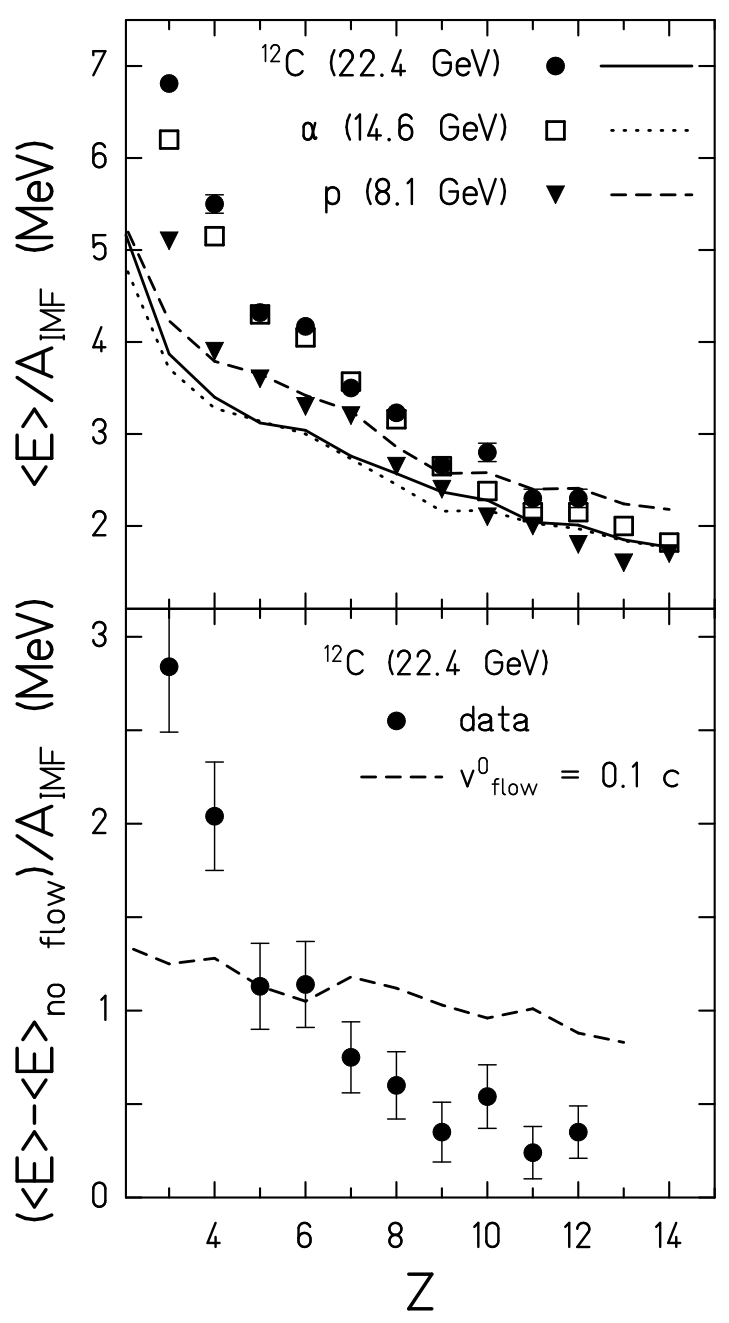

Fig. 2. Upper part: The mean kinetic energies of outgoing fragments per nucleon measured at $\theta=89^{\circ}$ for $\mathrm{p}(8.1 \mathrm{GeV}),{ }^{4} \mathrm{He}(14.6 \mathrm{GeV})$ and ${ }^{12} \mathrm{C}(22.4 \mathrm{GeV})$ collisions with $\mathrm{Au}$. The shown error bars are due to statistics only, a systematic error of $5 \%$ has to be added. The lines are calculated using INC+Expansion+SMM approach assuming no flow. Lower part: Flow energy per nucleon (solid points) obtained as a difference of the measured fragment kinetic energies and the values calculated without flow. The dashed line shows the calculations (see text) assuming a linear radial profile of the expansion velocity with $v_{\text {flow }}^{0}=0.1 c$ (at the surface). 


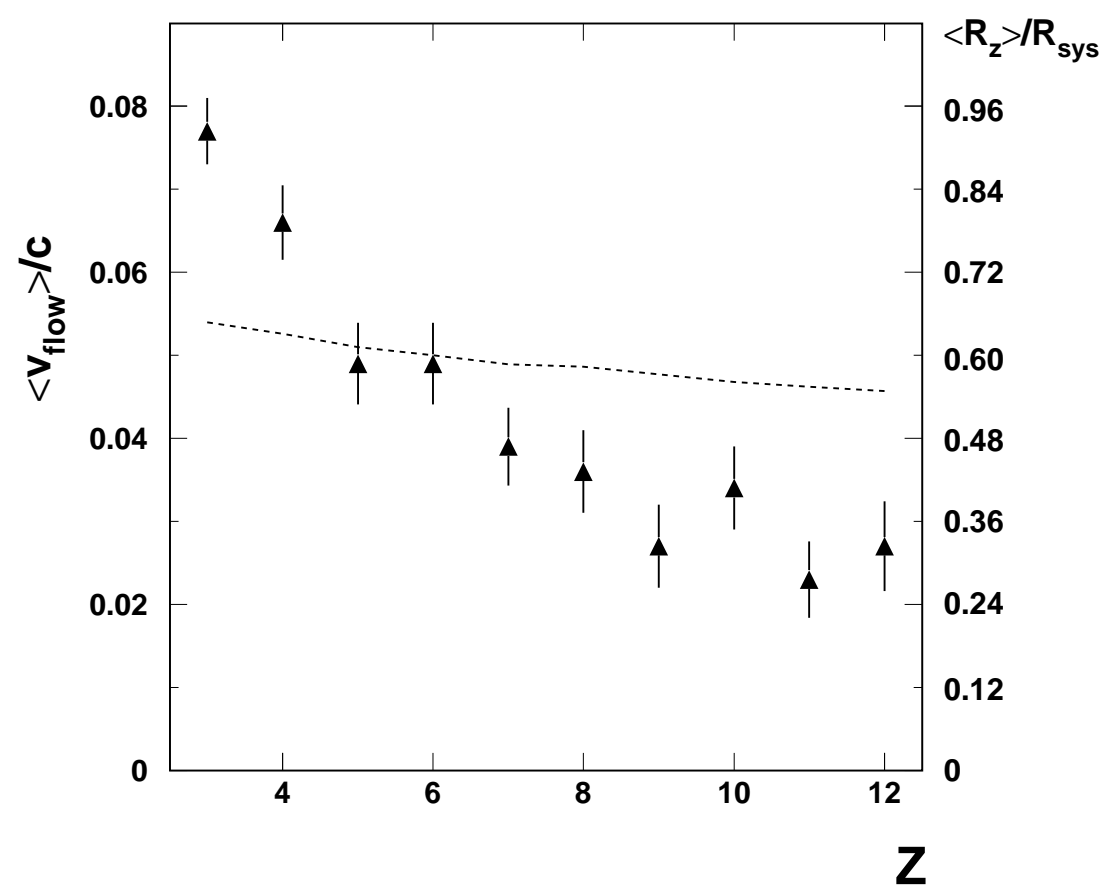

Fig. 3. Experimentally deduced mean flow velocities $\left\langle v_{\text {flow }}\right\rangle / c$ (left scale) for ${ }^{12} \mathrm{C}+\mathrm{Au}$ collisions (triangles) as a function of the fragment charge, and the mean relative radial coordinates of fragments $\left\langle R_{Z}\right\rangle / R_{\text {sys }}$ (right scale), obtained under the assumption of a linear radial profile of the expansion velocity. 